INTERNATIONAL DESIGN CONFERENCE - DESIGN 2018

https://doi.org/10.21278/idc.2018.0279

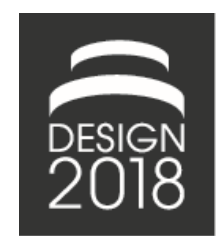

\title{
RETHINKING DESIGN EDUCATION FOR THE MULTICULTURAL GENERATION
}

\author{
T. Huang, A. Scott, Q. Lu and T. Sui
}

\begin{abstract}
The paper presents our strategies and insights for conducting a collaborative workshop of American and Chinese students and faculty in Guangzhou, China. Inspired by this experience, the authors address the challenges and opportunities of globalization and decolonization to design education. The paper emphasizes the importance of establishing strong collaborations with other countries and cultures for a truly multicultural experience for the new generations of design students.
\end{abstract}

Keywords: multi-/cross-/trans-disciplinary approaches, collaborative design, design education, design learning

\section{Introduction}

Discussions about change have never ceased in design education. Indeed, design educators feel the constant pressure to update their curriculum to adapt to the rapidly changing technology and culture (Findeli, 2001). Clearly, there are many ways to rethink design education. Our paper focuses on one aspect that we found crucial for the success of any design programs moving forward: we need to rethink our design education for the multicultural generation. As Yim et al. point out (2011), students should be able to appreciate the differences in cultures, and be ready to use such understanding for successful global collaboration in this age of globalization. More importantly, how do we build our core design education identity in a world of competing ideologies and cultural values? How do we take advantage of the global and multicultural background of our student body to expand their understanding of design?

Education itself is also becoming more and more global. This phenomenon presents both opportunities and challenges for higher education, especially design education, where cultural context usually plays a large role (Buchanan, 2004). Undoubtedly, this is not a new challenge in design education, but one that has always been one-sided: design institutions in the western world have interacted with enthusiastic students from other less developed countries, but students from the West seldom have been given the opportunity to work with their counterparts in those traditionally less advanced countries. For instance, in recent years, the rate of educational exchange has been accelerating in China. According to China Scholarship Council, a unit under the Chinese Department of Education, in 2017, the Chinese government is planning to sponsor 3,500 visiting scholars to study aboard for three to six months. According to UNESCO's report, in 2011, there are approximately 650,000 students from mainland China studying abroad, with approximately $27.5 \%(179,000)$ headed to the US. However, there were only 80,000 international students who studied in China during the same period of time, that was over half of million in difference (UNESCO Institute of Statistics, 2014). It is clear that Chinese universities want more international students to come to study with their own 
students so they can benefit from the cultural exchange, instead of the unidirectional export of their talents to the developed countries.

We have observed great success of American design students who participated in study abroad programs in Nepal, Denmark, and England over the years. To our knowledge, though there are many American design educators visiting or working in China each year, and also short-term design programs run by American design schools in China such as the Create Change Forums organized by Art Centre College of Design, few American design programs have organized American students to study alongside with Chinese students.

We decided to bridge this gap: to create a meaningful educational experience for both the American students and the Chinese students. Over the Christmas of 2016, the authors from Southern Illinois University Carbondale (SIUC) were invited by the College of Art and Design at Guangdong University of Technology (GDUT) to hold a week-long workshop with 28 Chinese students and four American students in Guangzhou, China. The workshop marked the first time such a workshop has been taught and participated in by American faculty and students at GDUT. It provided a rare opportunity for everyone involved to see first-hand the differences in pedagogy, design thinking, process, and methods between two design education systems.

Using this unique experience, and based on our two decades of combined experience teaching in design programs both in American and Chinese universities, we have developed several tangible ideas about how to rethink design education for the multicultural generation. In this paper, we will discuss our observations and analysis from several perspectives, from the planning of the workshop, to the actual outcome of the educational experience, from the logistics of the organization, to the future plan of similar projects.

\section{The educational challenge}

\subsection{The research question}

Our research questions for this project is: how does a short-term workshop provide a uniquely collaborative educational and cultural experience for students?

We used various artefacts created in the workshop and collected student feedback to answer this question. By answering it, we intended to set an example for similar endeavour for both American and Chinese universities in the future.

\subsection{The planning}

From June to December, 2016, the workshop took nearly six months to plan. While reviewing previous literature, we discovered a few precedents that discuss design courses participated by both Eastern and Western students in a university setting. For instance, Yim et al. (2011) present their findings from a collaborative project between German and South Korean students. They conclude that managerial, interdisciplinary, intercultural, team collaboration, and student preparation and technical issues are five aspects that must be considered when conducting such a design course. These were also the issues we planned for at the beginning of the collaboration, with a few additional challenges: we had only one week of time to conduct the workshop, and we must work with students from four different universities. In addition, unlike some of the similar programs conducted previously such as the East-Asian collaborative program described by Kim et al. (2009), the American educators did not know the Chinese participants in advance so we were not able to arrange teams according to students' "personal creative modes".

After much discussion, we decided that our planning should be based on these principles:

\section{Maximizing the diversity in disciplines}

First of all, the participants of the workshop was intentionally assembled to maximize the diversity in disciplines. GDUT organized both graduate and undergraduate students from its own industrial design program to participate, while also inviting graduate students from South China University of Technology (SCUT) and Guangzhou Academy of Fine Arts (GAFA). The three universities are vastly 
different both in their cultures and academic offerings, GDUT and SCUT are both comprehensive universities with a focus on technology, while GAFA is a renowned art school. All of them, nonetheless, are highly regarded for their industrial design program.

\section{Focusing on research and presentation skills}

Because SIUC's graduate program had not started at the time, their participants were senior and junior undergraduate students. Clearly, for this mixed audience, the theme of the workshop cannot too narrowly focus on skill building, but rather, should focus on research and presentation skills that are universal.

\section{Maximizing the diversity in educational experience}

The educators planned the educational exercises to be diverse and different from what the students were used to, especially in a Chinese university setting. We planned for two ice-breaker activities on the first day of class, both required students' active participation and presentation. In particular, we found it important to counter the problem of a lack of individual attention due to over-crowded classrooms in Chinese educational institutions (Huang, 2009). We dedicated two half-days to two mid-class group critique and reviews in which we insisted that every student must talk about their role and work in the project. It was of great importance to us that each member of the team had the opportunity to present to the audience. Individual presentations strengthens the students' sense of ownership to their team projects. We also recognized that this intense critique process is uncommon in Chinese classrooms, especially in non-art schools. Therefore, we wanted to continue this essential tradition in American design studio classes in our workshop so the students from China might have a genuine experience.

\subsection{The theme and deliverables}

Meanwhile, GDUT expected tangible results. Faculty from SIUC and GDUT jointly decided to set the theme of the workshop to be "Living in 2020", which left enough room for students to explore creative solutions while at the same time prevented them from going overboard with solutions that rely on completely unrealistic future technology.

Within the theme, the faculty further identified four possible scenarios to help students find common experience to understand and work from since they were complete strangers from two different countries at the beginning of the workshop. The four scenarios were:

- How does a traveller do laundry?

- How does a foreign traveller go from the airport to their hotel?

- How does a foreign traveller find help in a medical emergency?

- How does a foreign traveller order food?

\subsection{The implementation}

\subsubsection{The workshop format}

The format of the workshop was planned to be five days of studio where instruction and critique were given, with one additional half day for presentation and another two days for preparing and installing the final public exhibition. The instruction time was planned to be from 9 to 5 , with one hour lunch break in between. It quickly became obvious that half of the in-class time should be planned to be work time to allow students to work together to produce deliverables. Therefore, lecturing was completed on the third day and we devoted the remaining of the workshop to group work and critique.

\subsubsection{The team}

Each of the four American students was assembled into a team with seven Chinese students to ensure that each team had a mixture of talents, language abilities, and personalities. We conducted two icebreaker activities on the first day of class, which consumed half of the day but proved to be great exercises for team building. The Chinese and American students had meals together every day. The teams also interacted constantly outside of the workshop via social media. 


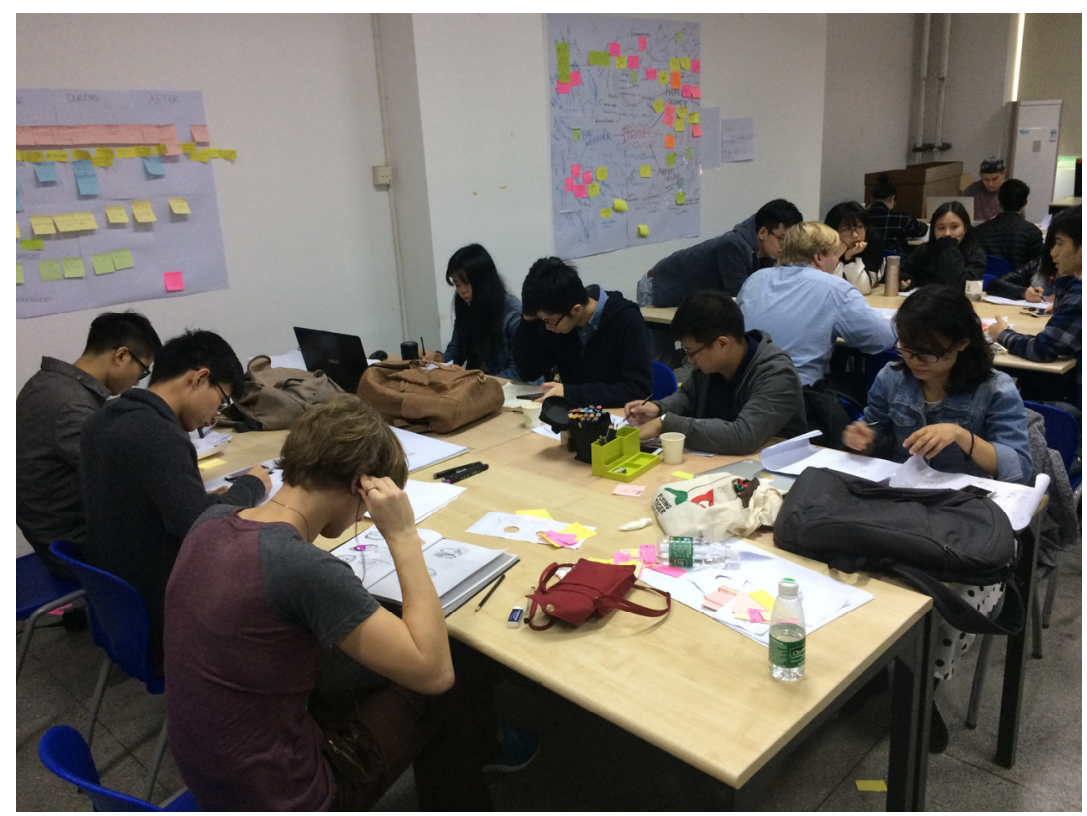

Figure 1. Group work activity, day 3

\subsubsection{The language barrier}

As mentioned by other educators conducting similar projects, the language barrier was a great challenge for any cultural exchange (Ebbert, 2016). To maintain the class's pace, we decided to conduct the workshop mostly in English. Our expectation was that since the graduate students in China have to pass a national English exam to enter the graduate schools, they must reach a certain listening and comprehension level of English in order to do so. However, these abilities varied greatly in individuals and many of our Chinese participants found it difficult to follow the lectures. It would have been a much different workshop had it not been for the fact that one of the American faculty was a native Chinese speaker, and the Chinese faculty from GDUT were able to provide assistance. Frequent translation was needed to ensure that students with lesser language ability could communicate effectively.

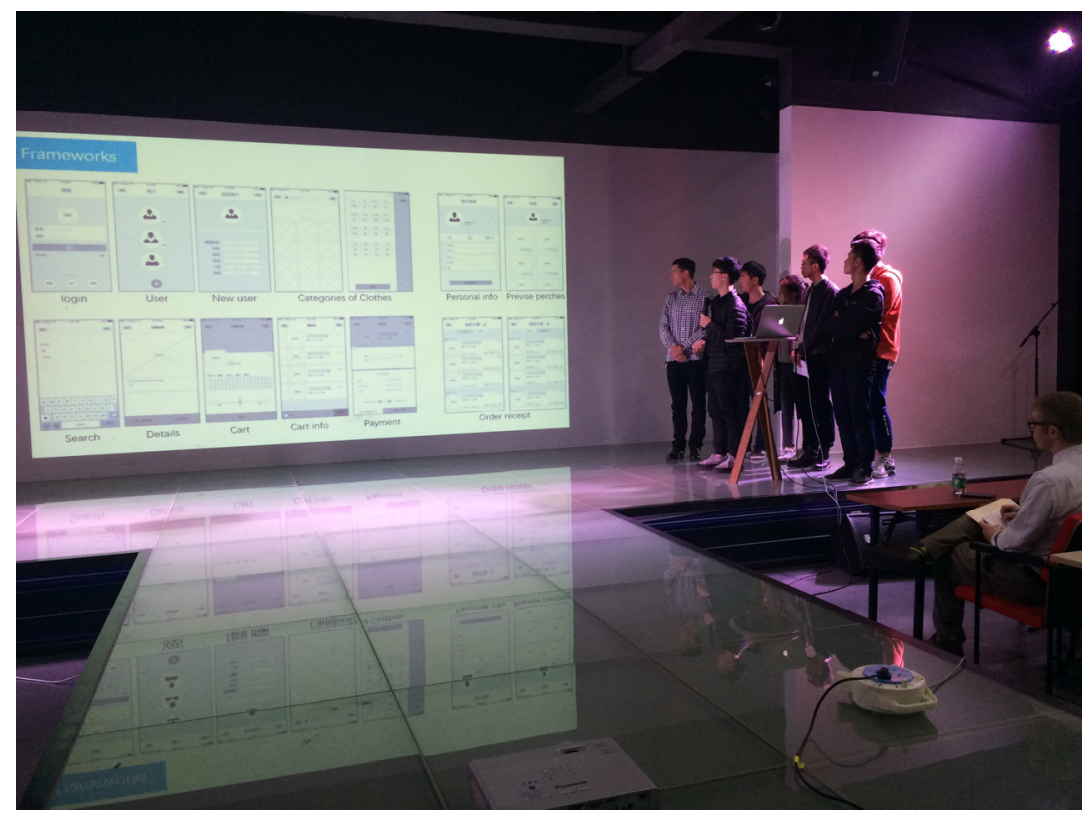

Figure 2. Final presentations, day 6 
The final presentation of the workshop was announced to the general public using posters and WeChat (a popular mobile social media APP in China). The event took place in the design auditorium and over 50 students and faculty other than the participants of the workshop attended.

\subsection{The results}

\subsubsection{The deliverables}

The final deliverables for the workshop were three artefacts: a digital presentation and two posters for the exhibition from each team. We required that a clear research and design process be presented in these artefacts. Other than that, we did not set clear parameters as we expected the students at this level would know how to build a successful presentation, and the teams would divide the workload properly. In the end, the students employed many visualization methods, including short film based on the user scenarios, animations/short videos, 3D model renderings, and physical models to describe their solutions, far exceeding our expectations.

During the workshop, GDUT also required that students be divided into different groups according to their background to write short reflections for each day. The reflection essays must be over 500 words in length and included images taken from the day. These essays were published daily on the public platform of WeChat.

The four teams eventually chose three scenarios to work with. Two teams focused on laundry service for travellers, one team focused on traveling safety, while one team focused on food ordering. Below we selected two posters from two teams due to the limitation on the length of this paper.

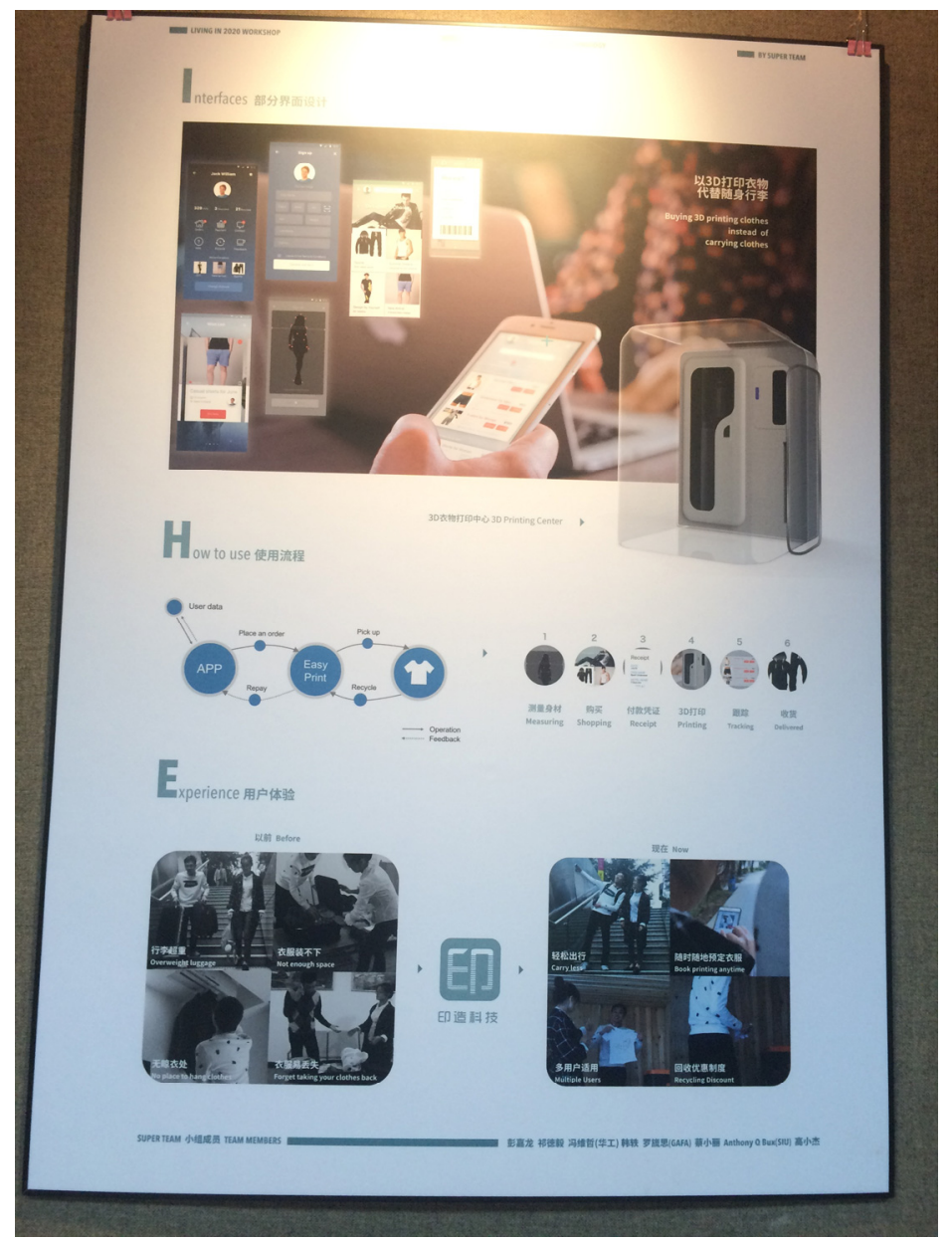

Figure 3. Laundry scenario poster 3, group YingZao (print/create) 


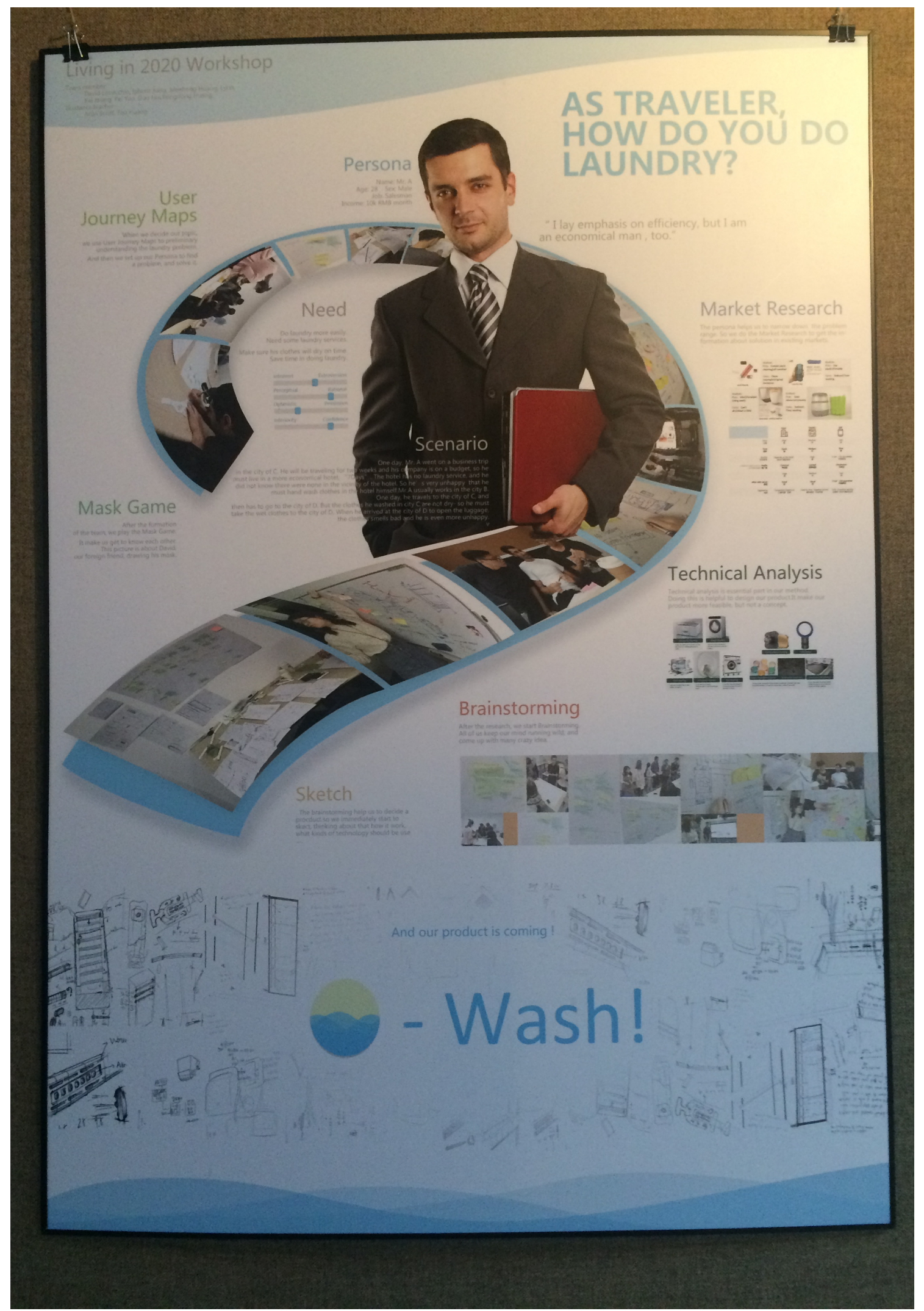

Figure 4. Laundry scenario poster 1, group O-Wash 


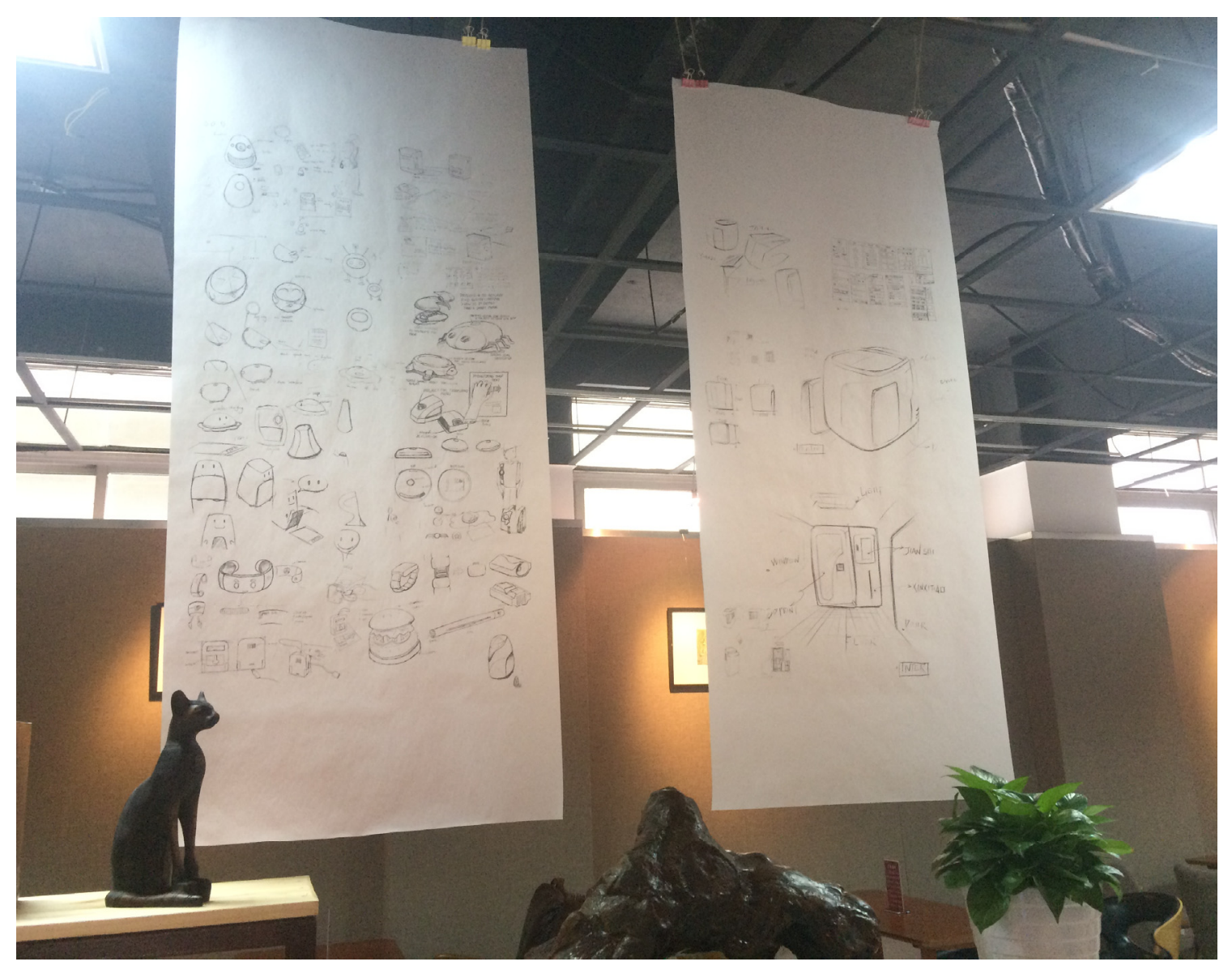

Figure 5. Sketches displayed in the exhibition

\subsection{The limitations}

Being an experiment, the workshop might not succeed in all of its goals. First of all, the original intent of the organizing faculty, was to involve students from various disciplines. However, while the three graduate students from the department of automation participated in the first half day of the workshop, they were so intimidated by the exercises and lectures that despite repeated reassurance from both Chinese and American faculty, they decided to quit. Therefore, we were unable to construct teams with different specialties as we originally envisioned, which we felt limited our solutions. Secondly, the timing of the workshop limited the participation of American students. Several factors determined the scheduling for this workshop, including the requirement of the grant that GDUT received to fund this workshop and the semester schedule of SIUC. The short amount of time for planning also limited the financial support that either universities could obtain. Finally, the content preparation and time management of the workshop could be improved based on student feedback.

\subsection{The future plan}

GDUT has organized the Chinese students to continue to perfect the solutions created in the workshop with the aim to use them for design competitions and commercialization. They have already applied and obtained two patents for one solution. GDUT and SIUC plan to continue our collaboration in this shortterm workshop format, or extend the workshop to be two to four weeks long to achieve better results. Faculty from SIUC also plan to replicate this workshop at more universities with different student populations in China to improve the course. 


\subsection{The evaluation}

\subsubsection{Artefacts}

As shown above, the deliverables created by the students were of high quality and made by a great variety of visual methods. These could not have been achieved without the concerted efforts from the majority of the students. These final outcomes showed that the workshop effectively brought the participants together towards a common goal in a short period of time, despite the social and language barriers. Judging by the outcomes, the collaboration and the educational program could be considered successful.

\subsubsection{Student reflections}

We considered student feedback an important part when evaluating the workshop. Both Chinese and American students expressed satisfaction with the workshop experience. The quotes below were selected and translated from the Chinese students' reflection essays:

"The most interesting thing about this workshop is that people from different schools and different countries can share their ideas."- Students from South China University of Technology

"When we were asked to present in English, we were worried at first. But we realized that was a good opportunity for us to practice our language skills. Fortunately, we had Professor Huang, who was able to translate for us every now and then."- Students from GDUT

The American students commented on the language barriers, group dynamics, and cultural insights that they obtained through this workshop as quoted below:

"Having this opportunity to come to Guangzhou for the 2020 Workshop has been a wonderful experience for us. It was the first time, we have ever collaborated with so many different kinds of designers in a group project. The sense of teamwork and camaraderie we have been observing and participating in is very inspiring to our work ethic. Adapting to new methods of communication has been an eye-opening experience and has taught us just how important communication is. Through word, gesture and a pen-we have broken the barrier and come up with concepts we are very excited about!" -David Lovecchio

"In my group we had two undergrads and six graduate students with a verity of different skills. We were all able to work together, to solve the problem of laundry of a traveller coming to Guangzhou. Even though we had a big language barrier between English and Chinese, we were able to overcome that from our understanding of design process and sketching... My group gave me a lot of insight of the culture of Guangzhou and how they normally to laundry and how much different it is in the U.S. ... Overall I had a great experience with my team members and the opportunity this workshop gave me."Anthony Bux

The main negative comments on the workshop was the tremendous pressure of completing the projects within only a week.

\section{Conclusions: Actionable findings}

We have observed and learned from this rare opportunity several insights to help answer our research question. Here are a list of these actionable findings for other academics and educators who are interested in planning similar programs.

\subsection{Actionable finding 1: Bilateral collaboration is essential}

These collaborations must be mutually beneficial to all institutions involved. Multiculturalism means that education programs involving faculty and students from vastly different cultural backgrounds should be exchanging ideas. As faculty from the West, we must recognize that we are not merely "bringing" our knowledge to the less developed countries. We must treat our counterparts in the other countries as equals, who understand their students' needs far better than we do. This is an excellent opportunity for everyone involved to examine our bias and gain a deeper understanding of design and education. 


\subsection{Actionable finding 2: Identify the core values of what each respective program can offer}

Conducting this workshop is part of our continuous effort to create values for our students. Because this educational experiment also made one issue more urgent and clear to us: how does an educational institution establish its core identity in this fast shrinking world? How does it maintain its uniqueness while embracing the multicultural background of our students? With the exception of a handful of elite educational institutions, the majority of educational institutions in the world, especially those traditional brick-and-mortar types, are facing this problem. Solving this problem needs design thinking, and collaboration across institutions.

\subsection{Actionable finding 3: Develop an effective way to communicate}

In this day and age, design skills and knowledge can be learned through multiple channels and do not necessarily need to be taught or transferred in a classroom anymore (Norman, 2014). The technical and physical platform we utilize to reach our audience will continuously be changing too (Rhodes, 2001). Interestingly, this makes multicultural interaction in education an even more important issue. Because though students from different cultural backgrounds might have similar access to technology, they are exposed to very different physical, cultural and social environments. Therefore, they choose to access very different information with little self-awareness. Digital media flattens the world, makes cultures in other places much more accessible, but it also flattens interactions. Yet, education is based on interaction, the interaction of people, and of people and their surroundings. Our interaction with the world becomes more extensive because of the development of technology and new communication processes, as well as the rapid increase of mobility of people in the past few decades. But the richness and subtly of human interactions are often lost in our daily saturation of information. The pinches and punches of our fingers on glass screens or keyboards cannot replicate all that a face-to-face experience can offer. As we observed, face to face interactions are still far superior to any other means to communicate. However, face to face interaction remains the most expensive form of education. Therefore, we must start the planning of these multicultural educational programs early to ensure that when it is finally time to meet, the experience will be fulfilling.

\subsection{Beyond an educational experiment}

In retrospective, the workshop was an educational experiment at many levels. Don Norman stated that modern design is not done in isolation and design education must change to allow designers to work with others (Norman, 2011). Indeed, global collaboration of design teams has been a reality for many years, yet our educational institutions have not been able to recreate these experiences in our classrooms due to multiple restrictions, especially in the developing countries. Our workshop has proven that we can mirror this aspect of the professional world in an educational environment with careful planning and perseverance, as well as with the right team. Design education programs that give their students the opportunity to work towards a common goal in a short period of time while navigating cultural differences are important for them to unveil their hidden preconceptions, and in this process, become better designers and global citizens. That is why we invite educators from around the world to create these enjoyable and meaningful programs, which will have a life-long impact on the young minds.

\section{Acknowledgement}

Special thanks to faculty and students from Guangdong University of Technology, especially Dr. Wen Jiang, Professor Yong Zhang, Professor Yibing Chen. We also want to thank Southern Illinois University Carbondale, Guangzhou Academy of Fine Arts, South China University of Technology for their support.

\section{References}

Buchanan, R. (2004), "Human-centered design: changing perspectives on design education in the East and West", Design Issues, Vol. 20 No. 1, pp. 30-39. https://doi.org/10.1162/074793604772933748 
Ebbert, C. (2016), "Collaboration issues in ethnically mixed student teams", The 18th International Conference on Engineering and Product Design Education, Aalborg, Denmark, September 8-9, 2016, The Design Society and The Institution of Engineering Designers, London, UK, pp. 676-681.

Findeli, A. (2001), "Rethinking design education for the 21 st century: theoretical, methodological, and ethical discussion”, Design Issues, Vol. 17 No.1, pp. 5-17. https://doi.org/10.1162/07479360152103796

Huang, T. (2009), Reforming Industrial Design Education in Mainland China: For Sustainability, VDM Verlag, Saarbrücken.

Kim, Y.S., Kim, M., Kim, K., Lee, S., Lee, S.W. and Kang, M. (2009), "Interdisciplinary product design and EastAsian collaborative design education program", The 17th International Conference on Engineering Design, (ICED 09), Vol. 10, Palo Alto, CA, USA, August 24-27, 2009, The Design Society, London, UK, pp. 289-300.

Norman, D. (2011), Design Education: Brilliance without Substance. [online] Available at: http://www.jnd.org/dn.mss/design_education_br.html (accessed 27.08.2011).

Norman, D. (2014), State of Design: How Design Education Must Change. [online] Available at: http://www.jnd.org/dn.mss/state_of_design_how.html (accessed 25.03.2014).

Rhodes, F.H.T. (2001), The Creation of the Future: the Role of the American University, Cornell University Press, Ithaca, NY.

UNESCO Institute of Statistics (2014), Higher Education in Asia: Expanding Out, Expanding Up. [online] Available at: http://www.uis.unesco.org/Library/Documents/higher-education-asia-graduate-universityresearch-2014-en.pdf (accessed 08.12.2017).

Yim, H., Lee, K., Brezing, A., Lower, M., and Feldhusen, J. (2011), "Learning from an interdisciplinary and intercultural project-based design course", Proceedings of E\&PDE 2011 / The 13th International Conference on Engineering and Product Design Education, September 8-9, 2011, The Design Society, London, UK, pp. $752-757$.

Dr. Tao Huang, Assistant Professor

Southern Illinois University at Carbondale, School of Art and Design

School of Art and Design, Southern Illinois University, 62901 Carbondale, United States

Email: thuang@siu.edu 